\title{
Danza Abend: \\ un grupo independiente fundado por la maestra Cristina Gigirey
}

Gabriela Dorries-Gigirey

Danza Abend

Bailarina y coreógrafa

Universidad Nacional

Costa Rica

Recibido: 17 de julio del 2019

Aceptado: 20 de diciembre de 2019

DOI: https://doi.org/10.15359/tdna.36-67.8

\section{Resumen}

Los inicios y el desarrollo de Danza Abend son inseparables de la práctica y enseñanza artística de la maestra Cristina Gigirey. No es posible referirse a Abend sin hablar de Gigirey, desde sus inicios en su Uruguay natal, y de cómo llegó a Costa Rica, donde su trayectoria artística, su práctica y su enseñanza son legados que persisten a lo largo del tiempo. A continuación, se presenta un itinerario de esta agrupación artística que recoge los grandes hitos de la experimentación y la práctica en la danza costarricense y de proyección latinoamericana.

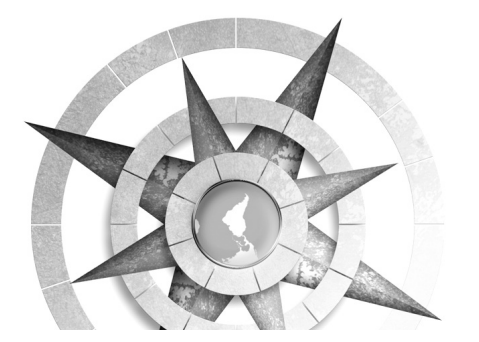

Palabras clave: Danza Abend, Cristina Gigirey, danza moderna, arte en América Latina
Abstract
The beginnings and evolution of Danza Abend are inseparable from the artistic practice and teaching of the teacher Cristina Gigirey. It is im- possible to refer to Abend without mentioning Gigirey, from her origins in her motherland Uruguay, and how she came to Costa Rica, where her artistic career, practice and teaching are legacies that remain over time. The following is an itinerary of this artistic group that gathers the major milestones of experimental and performing Costa $\mathrm{Ri}$ - can dance which had, as well, Latin American projection.

Keywords: Danza Abend, Cristina Gigirey, modern dance, arts in Latin America 


\section{Resumo}

O início e a evolução de Danza Abend são inseparáveis da prática artística e do ensino da professora Cristina Gigirey. É impossível referir-se a Abend sem mencionar Gigirey, desde as suas origens na sua pátria Uruguai, e como chegou à Costa Rica, onde a sua carreira artística, prática e ensino são legados que permanecem ao longo do tempo. Segue-se um itinerário deste grupo artístico que reúne os principais marcos da dança experimental e performativa costa-riquenha que teve, também, projecção latino-americana.
Palavras chave: Danza Abend, Cristina Gigirey, dança moderna, arte latinoamericano

\section{Breve esbozo: los inicios}

Cristina nace el 6 de noviembre en el año 1940 en Montevideo, Uruguay y fallece el 5 de diciembre del 2006, en San José, Costa Rica a los sesenta y seis años de edad. Nace en el hogar formado por Delia Nadal y Luis Gigirey, ambos maestros de escuela, amantes de la cultura, el arte y la paz, cualidades que le inculcaron desde muy pequeña a su hija.

Fotografía 1.

Gabriela Dorries.

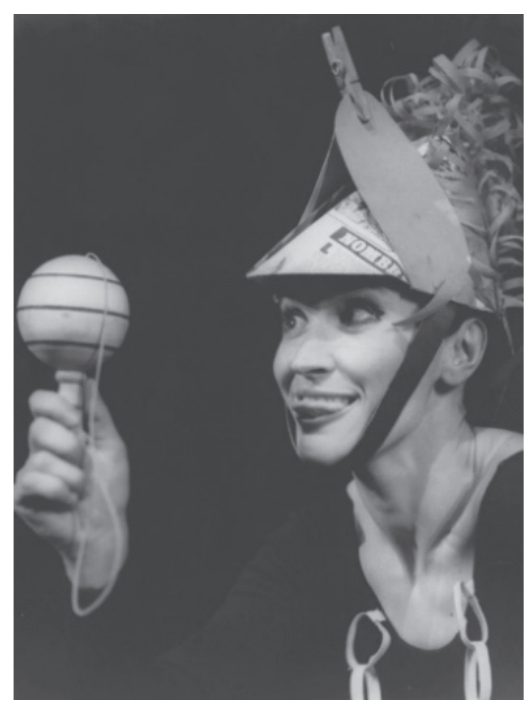

Fuente: Archivo fotográfico de Gabriela Dorries (s.f).

\section{(6)}

122 Danza Abend un grupo independiente fundado por la maestra Cristina Gigirey Gabriela Dorries-Gigirey 
Sus estudios de ballet se iniciaron a temprana edad y se prolongaron hasta su adolescencia, cuando comenzó a interesarse también por la danza contemporánea. Gigirey se inició en el Ballet de Cámara de Montevideo, dirigido por Hebe Rosa, con el cual incursionó en obras de corte más contemporáneo, pero sin dejar la técnica clásica. Ella decía con frecuencia sobre esa época que se descalzó para bailar, pues ya no bailaba en zapatillas de punta. Bajo la tutela de la maestra Rosa, Cristina bailó varias coreografías que incluso fueron vistas por el coreógrafo José Limón.

Fotografía 2.

Cristina Gigirey.

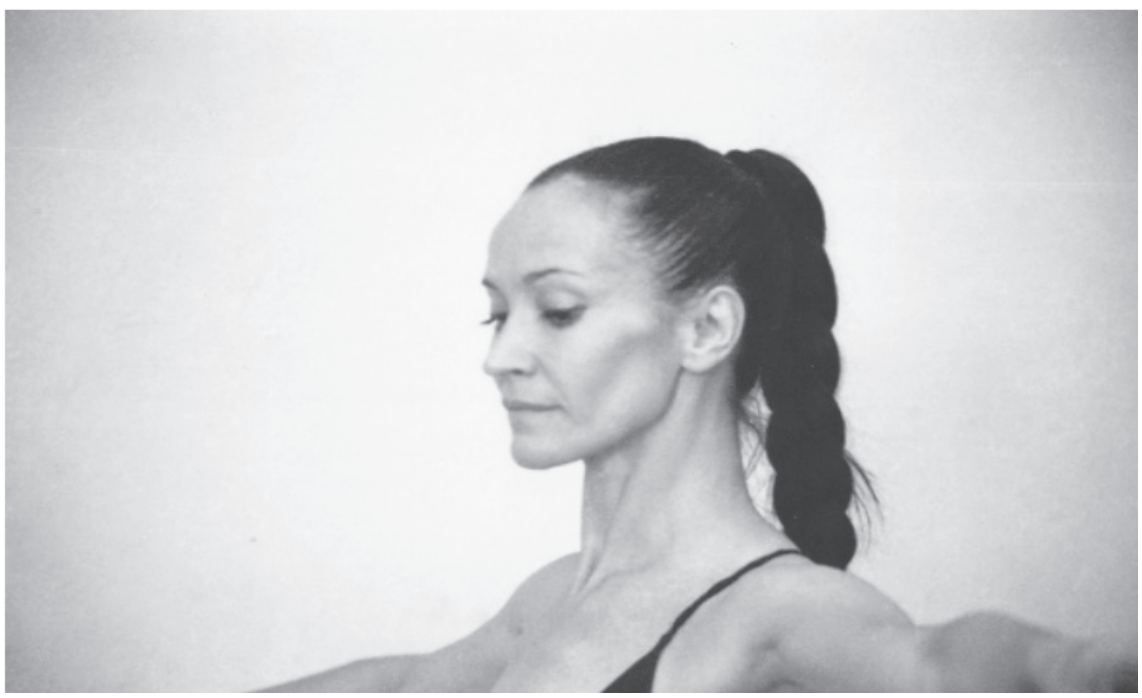

Fuente: Archivo fotográfico de Gabriela Dorries (s.f)

En 1963 fue invitada por el Ballet Nacional de Chile. Ahí tuvo su primer encuentro con la corriente impresionista alemana, cuando pudo trabajar con Patricio Bunster, Joan Turner y Sigurd Leeder. Regresa a Montevideo después de un año en Chile y como resultado de una beca concedida a su esposo, viajó en 1968 a Alemania, en donde estudió y montó coreografías, dio clases y bailó por todo el país. Trabajó con maestros de renombre como Yvonne Georgi, Palucca, Walter Nicks, John Cranko, Yuriko, entre otros.

\section{(C) $\odot \Theta \Theta$

SinDerivadas 3.0 Costa Rica 


\section{Su llegada a Costa Rica}

La década de 1970 fue muy rica para los costarricenses, ya que por los acontecimientos políticos ocurridos en varios países suramericanos, principalmente Chile, Argentina y Uruguay, una rica migración de talentos de diferentes campos artísticos arribó al país. La pre- sencia, acción y creatividad de estos exilados contribuyó a enriquecer el campo cultural costarricense.

En 1971 llegan a Costa Rica una serie de músicos de Europa, América del Sur y Estados Unidos, para conformar la Orquesta Sinfónica Nacional.

Fotografía 3

Cristina Gigirey.

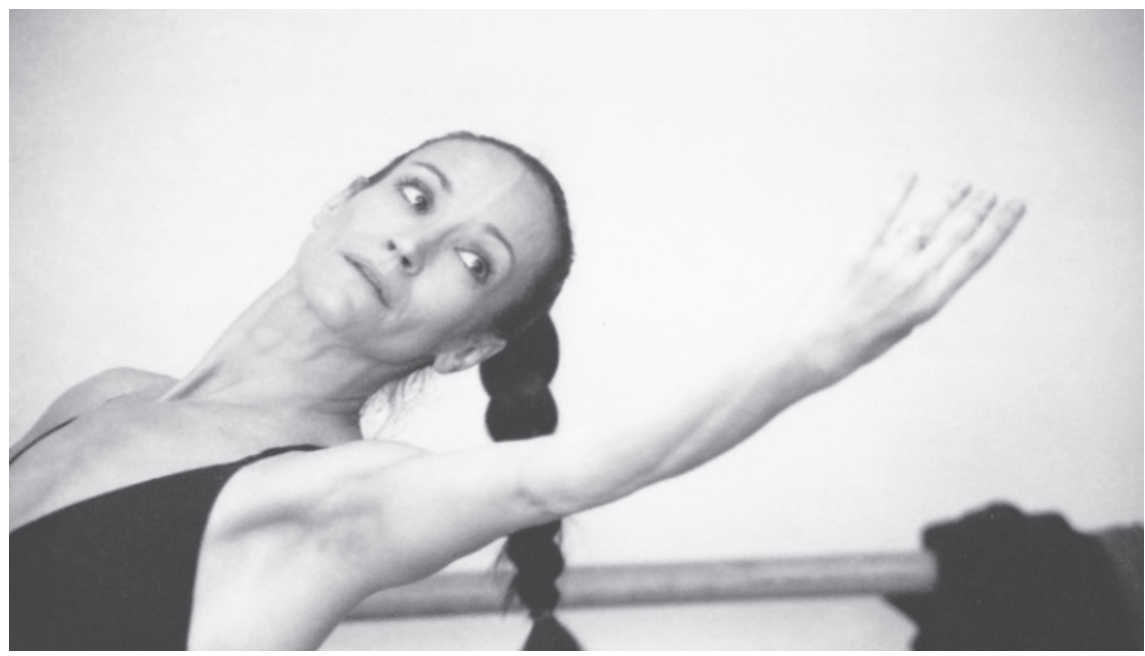

Fuente: Archivo fotográfico de Gabriela Dorries (s.f)

También la danza se vio beneficiada ampliamente con la presencia de figuras de gran relieve, talento, gran sensibilidad y creatividad, entre ellas es posible citar a la maestra, bailarina y coreógrafa Gigirey, quien arribó a Costa Rica en 1974. Cristina fue una de las profesoras fundadoras de la Escuela de Danza en la
Universidad Nacional, institución donde se desempeñó como profesora de Ballet Clásico por más de treinta años. Junto a la maestra Elena Gutiérrez, otra gran figura de la danza, fundó el Ballet Moderno de Cámara, del cual fue maestra y coreógrafa entre 1974 y el 1976.

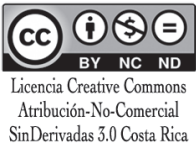

124 Danza Abend un grupo independiente fundado por la maestra Cristina Gigirey Gabriela Dorries-Gigirey 
Más adelante, al retirarse del Ballet Moderno de Cámara, la contactó el maestro Rogelio López y crean juntos el grupo Danza Universitaria. Con dicho grupo trabajó como coreógrafa y maestra de 1977 al 1982.

En 1980 Cristina montó su propio estudio de danza, labor que llevó paralelamente a su trabajo en la Universidad Nacional. Con el tiempo, al sentirse establecida en su estudio, decide crear un grupo independiente, y es así como nace, en 1983, Danza Abend.

\section{Fotografía 4}

Gabriela Dorries.

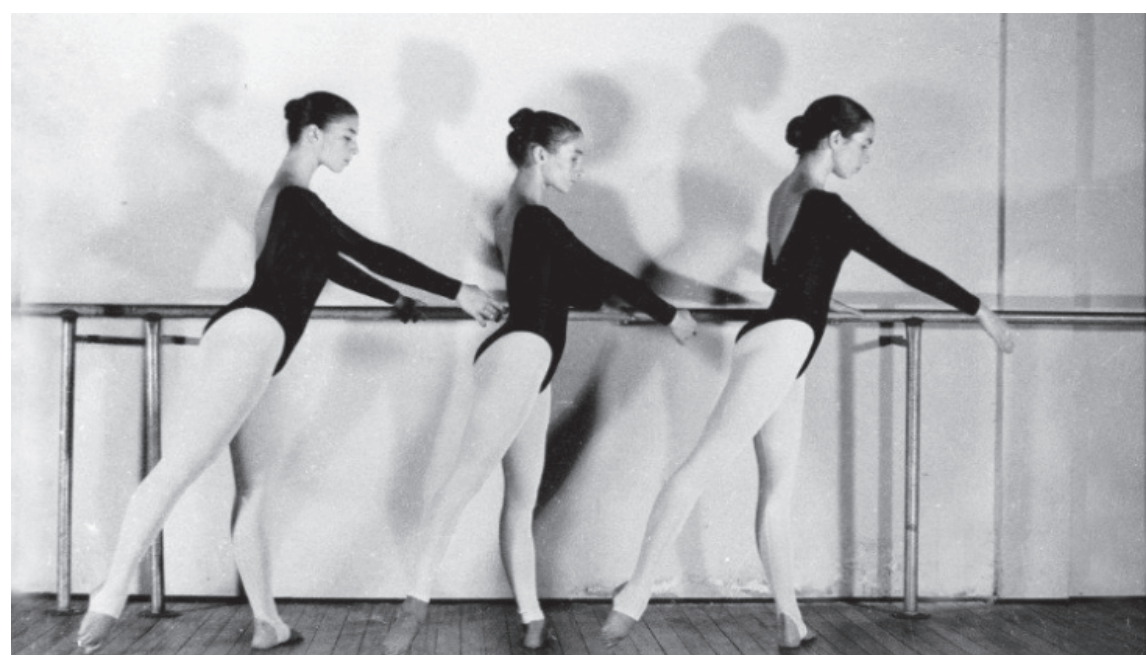

Fuente: Archivo fotográfico de Gabriela Dorries. (s.f)

\section{Danza Abend: los años 1980}

Cuando Cristina Gigirey creó y asumió la dirección artística del grupo Abend en 1983, inició un proyecto de danza independiente que hoy día se reconoce como un concepto con infraestructura sólida; entrenamiento, técnica y volumen interpretativo, un repertorio vigente, excelencia musical y continuidad y evolución. 
Gigirey, maestra indiscutible de bailarines profesionales y artistas en formación de todos los grupos existentes en aquel momento, fue una creadora que impulsó la evolución de la danza contemporánea en Costa Rica, desde su arribo en 1974. Bailarina de gran talento, siempre dijo que después de cada función no se sabía si habría otro espectáculo, porque todo empieza de nuevo al terminar la última función de cada temporada. Esta experiencia tan particular, asentada en el compromiso artístico personal del elenco y de su maestra y directora, ha generado durante todos estos años de Abend una opción renovadora, más que una compañía de danza.

Con Abend, Cristina realizó varios espectáculos en la década de los ochenta, los cuales denominó ABEND I, ABEND II y así sucesivamente hasta llegar al ABEND VI. No es desacertado asegurar que casi todos los bailarines de aquella época fueron formados y bailaron obras de Gigirey.

Durante el período, el grupo interpretó de forma intensa, dentro y fuera del país, obras como Las madres, Aquel soldado, Aquella mujer, Yerma, Páginas intimas, Los cuatro temperamentos, entre otras, que fueron creadas para Abend. Al mismo tiempo, Cristina retomó coreografías que había creado para las agrupaciones con las que había trabaja- do con anterioridad, como La casa de Bernarda Alba, Proceso, Aproximaciones 1,2,3, Danzas inglesas, entre otras.

Se puede decir con certeza que muchas de estas obras se fueron convirtiendo en repertorio importante de la historia de la danza nacional.

Danza Abend tuvo la oportunidad de representar a Costa Rica varias veces en el extranjero. Durante la década de 1980 estuvo muchas ocasiones en México, país que llegó a adoptar a Cristina con mucho cariño y con el cual ella mantuvo un gran vínculo durante toda su carrera. Quizá una de las más importantes participaciones fue cuando Danza Abend bailó en el Festival Cervantino en Guanajuato.

\section{El decenio de 1990}

A principios de los años noventa surgen varios cambios importantes para Danza Abend. Se crea una nueva agrupación, el Abend Juvenil, semillero que formó bailarinas desde muy temprana edad, las cuales fueron convirtiéndose luego en elenco estable del grupo. 


\section{Fotografía 5.}

La casa de Bernarda Alba. Coreógrafa de Cristina Gigirey.

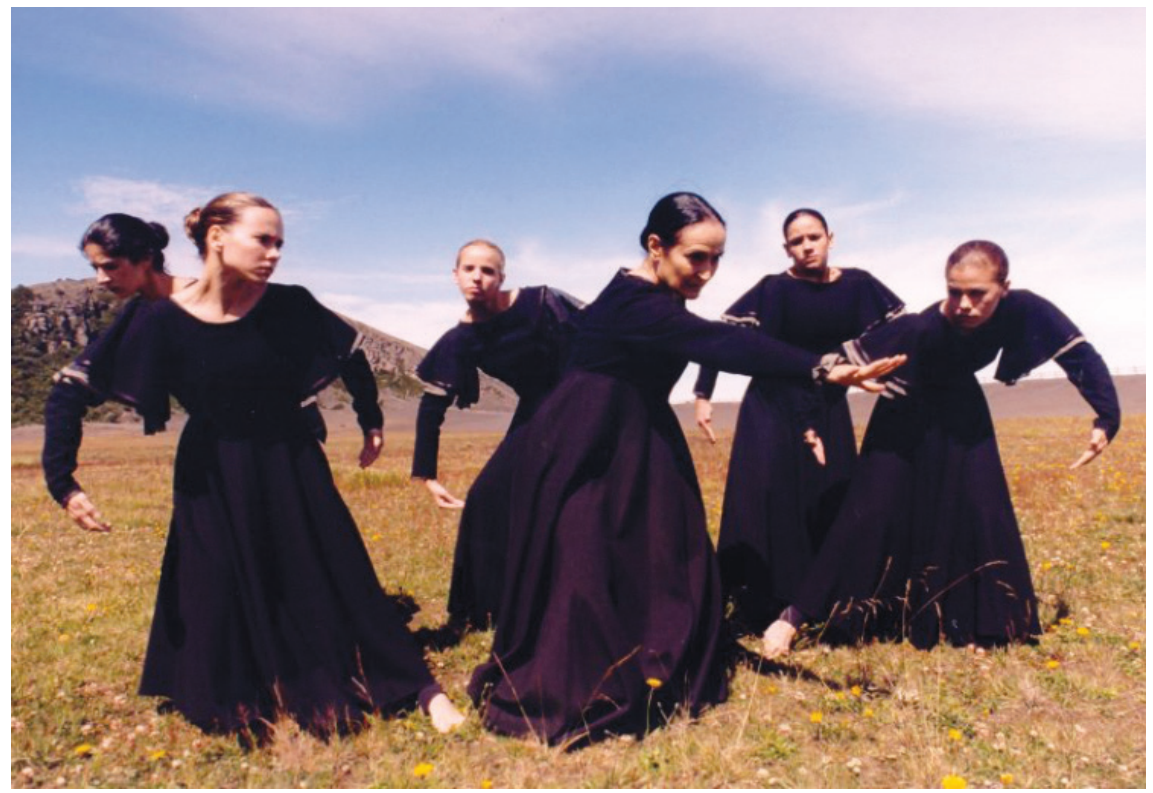

Fuente: Archivo fotográfico de Gabriela Dorries (s.f)

Anteriormente, Danza Abend había realizado un poco de extensión en las comunidades, pero es hasta el momento en que se crea el grupo juvenil cuando se comienza a hacer mucha más extensión con programas completos como Danza en la Iglesia y Danza en el Jardin. Estos proyectos se realizaron en varias provincias del territorio nacional y en diversas ocasiones. El primero buscaba bailar dentro de las iglesias como complemento, después de misa; y el segundo bailó en los jardines del Centro Cultural de México y en varios lugares al aire libre.
Se buscaron lugares no tradicionales para poder realizar espectáculos de danza, con la finalidad de alcanzar a un público que no tenía la posibilidad de ir a un teatro o que incluso no estaba interesado en ver danza. Mediante estas actividades se logró difundir un estilo de danza que emocionó, alegró, sensibilizó y, en fin, alcanzó a un público que no estaba acostumbrado a ver ningún espectáculo dancístico.

Con Danza Abend se realizaron varias giras de extensión de la mano de la Sinfónica Nacional, con lo cual se logró 
llegar también a comunidades que tenían tal vez la costumbre de oír a la orquesta, pero no de verla acompañada con danza.

Durante aquellos años también se hicieron intercambios con varios coreógrafos que fueron invitados por la maestra Gigirey a participar con su grupo. Figuras nacionales como Elena Gutiérrez, Rogelio López, Luis Piedra, Ileana Álvarez, Rolando Brenes, Jorge Ramírez, Marianela Vargas, entre otros y extranjeros como Jessica Fogel y Cora Flores fueron parte de este esfuerzo. Asimismo, se tuvo la oportunidad de bailar parte de estas obras compartiendo escenario con bailarines del calibre de Alexander Solano, Carolina Córdoba, Daniel Marenco, Humberto Canessa, Nandayure Harley, Oscar Chanis, Silvia Montero, Adrián Figueroa, entre otros.

A finales de la década del noventa comienza a surgir una inquietud entre las bailarinas del elenco estable de la Compañía. Querían comenzar a crear pequeñas obras y coreografías. Gigirey las amadrinó e incluso las incentivó para que lo realizaran, creando espacios para que ellas pudieran ensayar y una vez finalizado el producto lo pudieran presentar en escena.

\section{El período 2000 - 2006}

Entre el 2000 y el 2006 se continuó con la extensión universitaria, esta vez no solo bailando, sino montando obras de Gigirey en Liberia, provincia de Guanacaste, con bailarines de la zona, y también impartiendo talleres en San Ramón, provincia de Alajuela. Incluso UNA Danza Joven tuvo en repertorio por varios años dos obras de Cristina, Los Candombes y parte de Proceso y El Desubique. Con UNA Danza Joven también se realizó extensión.

Abend continuaba con las giras dentro y fuera del país, visitando Estados Unidos y México, donde se le otorgó a Cristina el Premio de Mujer del año 2000 y en donde participó en el homenaje que se le realizó a Cora Flores y Evangelina Villalón.

En el 2006, Abend, participa como uno de los pocos invitados internacionales al World Dance Alliance en Toronto, Canadá. En esta ocasión Cristina baila junto a su Abend La casa de Bernarda Alba, un éxito enorme y rotundo. Esta fue la última vez que bailó, pues Cristina Gigirey falleció aquel mismo año. Tenía sesenta y seis años. 
Fotografía 6.

World Dance Alliance, Toronto Canadá, 2006.

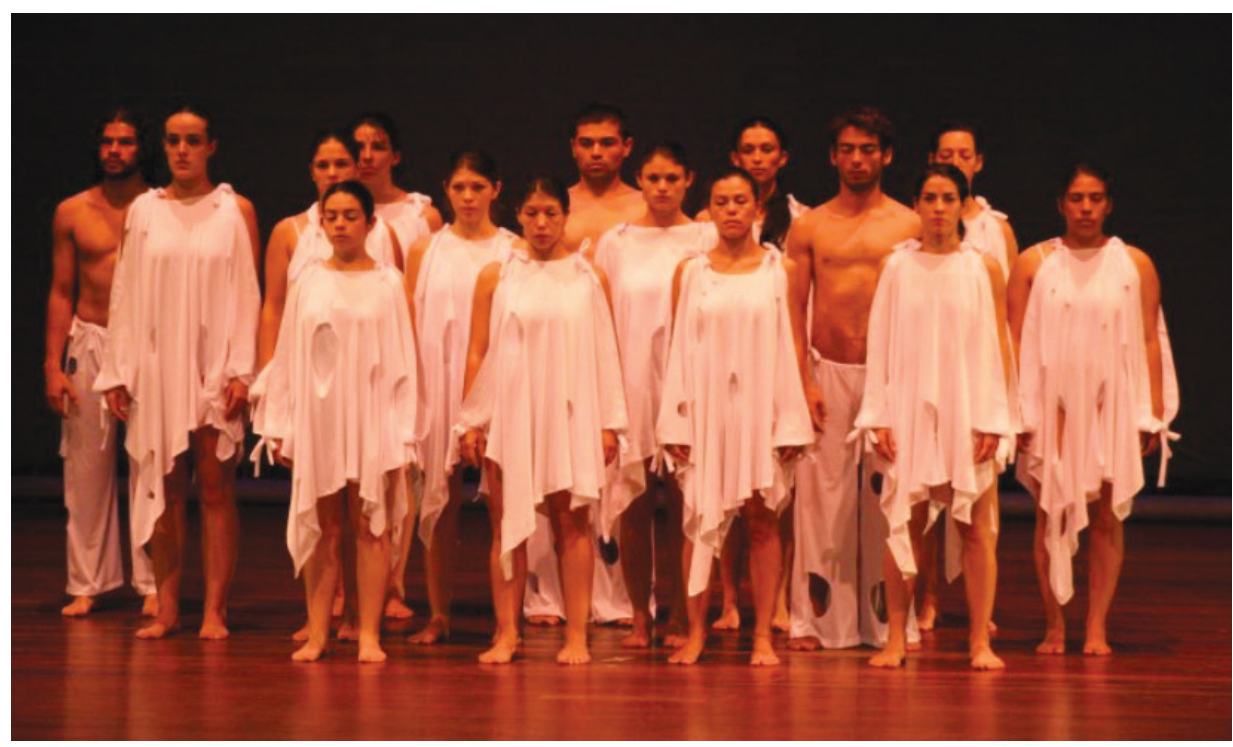

\section{Del 2006 a la}

\section{contemporaneidad}

Luego del fallecimiento de Gigirey, su estudio continuó funcionando bajo la dirección de su hija, Gabriela Dorries. En el 2010 se cierran las puertas del estudio, pero Dorries mantiene vivo el repertorio remontando obras con la Compañía Nacional de Danza, con
Danza Piazzolla, en Liberia, y con UNA Danza Joven, de la Universidad Nacional, entre otras.

Danza Abend puso en escena, a partir del 2006, obras de repertorio que llevaban muchos años de no ser vistas. Obras como Los cuatro temperamentos, Totentaz y Ostinato. 
Fotografía 7.

Los cuatro termperamentos, 2006

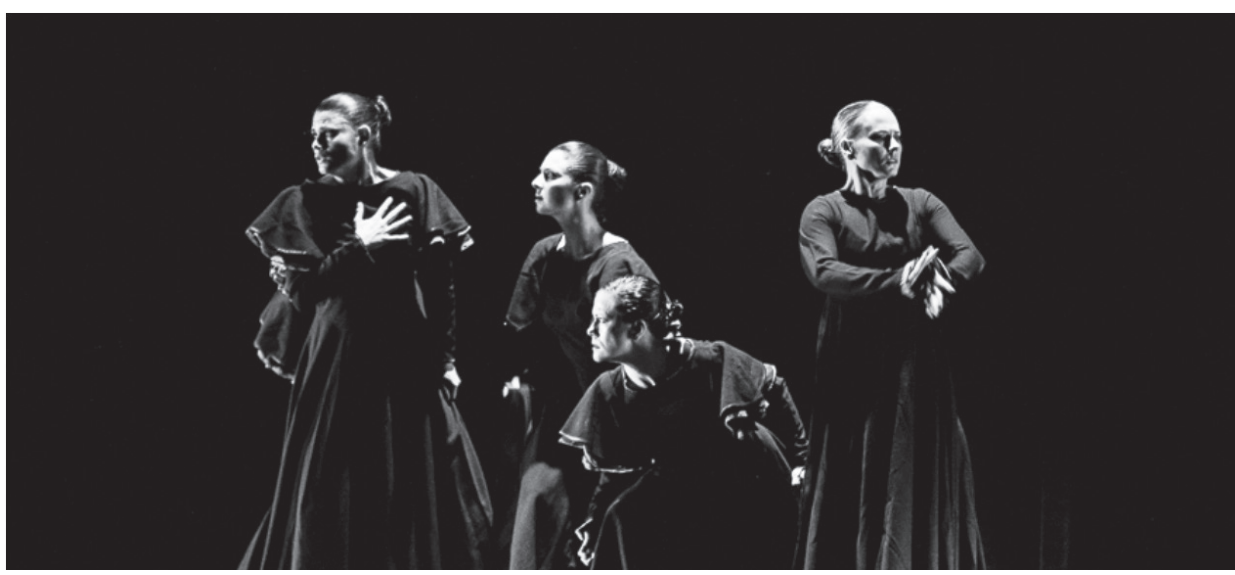

En el 2014 Danza Abend realizó un homenaje a Cristina dentro del marco del Festival de Coreógrafos del Teatro Nacional, en donde se volvieron a presentar La casa de Bernarda Alba y Proceso; y en 2015 retomó Aproximaciones 1, 2 ,3, junto con las obras anteriores, para presentarla en el proyecto Teatro al Mediodía del Teatro Nacional. En el primer semestre del 2016 se bailó Aproximaciones 1, 2, 3 en Nueva York, Estados Unidos y serán interpretadas por una costarricense y dos bailarinas norteamericanas.

Cristina siempre fue una excelente maestra, bailarina y coreógrafa. Fue fiel defensora del repertorio, de la buena técnica, de la danza. La mayoría de sus éxitos y de la continuidad de sus enseñanzas y el mantener vivo su repertorio fue gracias a Danza Abend, del cual ella siempre dijo que no era un grupo o una compañía, sino un concepto.

Con frecuencia decía: "Danza Abend es Cristina Gigirey". Hoy podemos decir que esa afirmación es verdadera, pero también podemos decir que a pesar de que ella ya no está con nosotros su legado prevalece a través de sus obras, sus enseñanzas, sus alumnos, sus bailarines y todo aquel que tuvo la oportunidad de trabajar con ella. 